\title{
A co-design for CAN-based networked control systems
}

\author{
Nguyen Trong Cac, Nguyen Van Khang
}

School of Electronics and Telecommunications, Hanoi University of Science and Technology, Hanoi, Vietnam

Email address:

cac.nguyentrong@hust.edu.vn (N. T. Cac), khang.nguyenvan1@hust.edu.vn (N. V. Khang)

\section{To cite this article:}

Nguyen Trong Cac, Nguyen Van Khang. A Co-Design for CAN-Based Networked Control Systems. Automation, Control and Intelligent Systems. Vol. 2, No. 1, 2014, pp. 6-15. doi: 10.11648/j.acis.20140201.12

\begin{abstract}
The goal of this paper is to consider a co-design approach between the controller of a process control application and the frame scheduling for CAN-Based Networked Control Systems in order to simultaneously improve the Quality of Control (QoC) of the process control and the Quality of Service (QoS) of the CAN-based network. First, we present a way to calculate the closed-loop communication time delay and we compensate this time delay using the pole-placement design method. Second, we propose a hybrid priority scheme for the message scheduling which allows to improve the QoS. Finally, we present a co-design of the communication time delay compensation and the message scheduling, which gives a more efficient Networked Control System.
\end{abstract}

Keywords: CAN Bus, Networked Control Systems, Message scheduling, Hybrid Priority Schemes, Communication Time Delay, Pole Placement Design, Co-Design

\section{Introduction}

The study and design of Networked Control Systems (NCSs) is a very important research area today because of its multidisciplinary aspect (Automatic Control, Computer Science, and Communication Network). The current objective of NCS design today is to consider a co-design in order to have an efficient control system [1,2]. Several works [2-6] have considered the co-design problems by combining control and scheduling messages. The works [2, 3] have considered the pole-placement design for time delay compensation and Large Error First (LEF) scheduling algorithm for message scheduling. The value of error is encoded directly into the priority. The higher the error is, the higher the message priority is and vice versa. A limitation is that, we have a wide range of the error value and this is not bounded (for example when the system is unstable, the error is infinite). Mapping these error values in the definite number of priority bits is not an easy task. Paper [4] has considered a hybrid priority scheme for message scheduling using the control signal $u$. The identifier field of the message which represents the message priority is divided into 2 small fields, the first represents a static priority and the second represents a dynamic priority. However, the limitation of this work is to only use 4 bits for static priority field, so they can determine a maximum number of 16 data flows (or nodes) which is not enough to address all nodes in a NCS. The work [5] only compensates the time delay from the controller to the actuator, not the closed-loop time delay. The work [6] considers the issue of co-design combines scheduling messages (static priority scheduling) and communication delay compensation.

From the above analysis, we found the same problem co-design of NCS design by combining control system (considering delay compensation issues) and communication networks (considering message scheduling problems) is a new field many investors and should be studied more. Therefore, in this paper will present the co-design of closed-loop time delay compensation and the message scheduling to improve QoC and QoS simultaneously and to have a more efficient NCS.

This paper includes the following sections: the section 2 presents the general context of the study; the section 3 presents the proposal for computation and compensation of closed-loop time delay; the section 4 presents the proposal of a hybrid priority scheme for message scheduling; the section 5 presents the co-design; the conclusion is represented in the section 6 .

\section{Context of the Study}

\subsection{Inverted Pendulum Application}

Structure diagram of an inverted pendulum mounted on a trolley is shown on Fig. 1.

The parameters are chosen as follows: the weight of the trolley $M=0.94 \mathrm{~kg}$, the trolley has the weight of $m=0.23 \mathrm{~kg}$ 
and the length of $l=0.3 \mathrm{~m}$, free fall acceleration $g=9.81 \mathrm{~m} / \mathrm{s}^{2}$, $\theta$ is the deviation angle of the pendulum, $x$ is the position of the vehicle, $u$ is the force putting into the trolley.

The purpose of the control problem is to move the trolley from position $x_{0}=0$ (initial position) to desired position $x_{1}=$ $0.1 \mathrm{~m}$ while keeping the pendulum vertical $\theta(t)=0$.

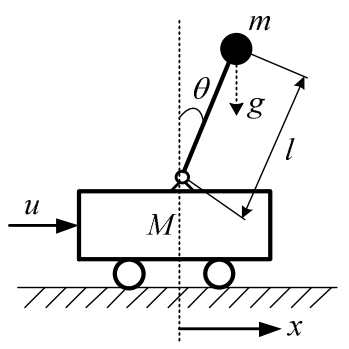

Fig 1. Structure diagram of an inverted pendulum mounted on a trolley.

The set point $x_{1}$ is a position echelon type applied at the time $t=0.5 \mathrm{~s}$. The desired control parameters include: damping coefficient $\zeta=0.707$, rise time $t_{r}=600 \mathrm{~ms}$, natural pulsation $\omega_{n}=1.8 / t_{r}=3 \mathrm{rad} / \mathrm{s}$.

State space model in continuous time [7]:

$$
\left\{\begin{array}{c}
\dot{x}=A x(t)+B u(t) \\
y(t)=C x(t)+D u(t)
\end{array}\right.
$$

Where:

$$
\begin{aligned}
& A=\left[\begin{array}{cccc}
0 & 1 & 0 & 0 \\
0 & 0 & -\frac{m g}{M} & 0 \\
0 & 0 & 0 & 1 \\
0 & 0 & \frac{(M+m) g}{M l} & 0
\end{array}\right], B=\left[\begin{array}{c}
0 \\
\frac{1}{M} \\
0 \\
-\frac{1}{M l}
\end{array}\right] \\
& C=\left[\begin{array}{llll}
1 & 0 & 0 & 0
\end{array}\right], D=0
\end{aligned}
$$

State-space model in discontinuous time domain with the sampling period $h$ is described as follows [7]:

$$
\left\{\begin{array}{c}
x(k h+h)=\Phi x(k h)+\Gamma u(k h) \\
y(k h)=C x(k h)+D u(k h)
\end{array}\right.
$$

Where:

$$
\begin{gathered}
\Phi=e^{A h} \\
\Gamma=\int_{0}^{h} e^{A s} d s B
\end{gathered}
$$

The discrete controller is:

$$
u(k h)=-K_{d} x(k h)
$$

The sampling period $h$ is defined by considering the following formula [7]:

$$
0.1 \leq \omega_{n} h \leq 0.6
$$

Where $\omega_{n}$ is the natural pulsation $(\mathrm{rad} / \mathrm{s})$. We choose the sampling period $h=50 \mathrm{~ms}$.

The state matrix $\Phi$ and $\Gamma$ are calculated by Equation (3) and (4). The state feedback matrix $K_{d}$ is calculated by using Ackerman function.

The performances of the discrete time system are as followed: the Overshoot of the angle $O=5.07 \%$, the setting time $t_{s}=0.4 \mathrm{~s}$, and the time responses are shown on Fig. 2 .

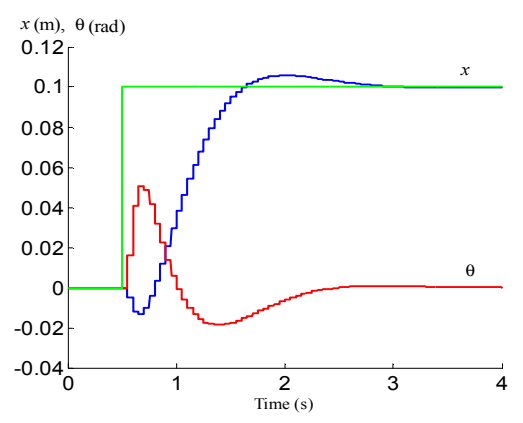

Fig 2. Time responses

\subsection{Implementation of a Process Control Application on a CAN Network}

The general model of the implementation of a process control application through a network is shown on Fig. 3.

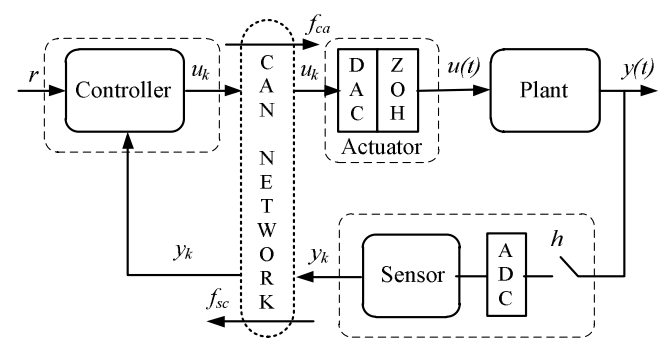

Fig 3. Implementation of a process control application on a CAN network.

We have three tasks: sensor task, controller task and actuator task. The sensor task is Time-Triggered while the controller task and the actuator tasks are Event-Triggered.

The sensor receives the sampled output $y_{k}$ provided by the Analog Digital Converter (ADC) and sends a frame including $y_{k}$ to the controller via the communication network. The controller receives $y_{k}$ from the sensor, then calculates the control signal $u_{k}$ and sends a frame including $u_{k}$ to the actuator via the communication network. The actuator receives $u_{k}$, converts $u_{k}$ into analog signal $(u(t))$ using the Digital Analog Converter (DAC) and then directly applies $u(t)$ to plant. The Zero Order Hold $(\mathrm{ZOH})$ keeps the value $u(t)$ until the reception of the next value.

We have two flows of frames: the Sensor-Controller flow $\left(f_{s c}\right)$ concerning the frames going from the sensor to the controller (denoted " $f_{s c}$ frame"), and the Controller-Actuator flow $\left(f_{c a}\right)$ concerning the frames going from the controller to the actuator (denoted " $f_{c a}$ frame"). 


\subsection{Communication Time Delay}

Communication time delays in each sampling period consist of 2 components:

- The time delay is due to transferring $f_{s c}$ frames (noted $\tau_{s c}$ ) which is the duration between the sampling instant $\left(t_{k}, k\right.$ $=0,1,2 \ldots)$ and the reception instant of this frame by the controller. The time delay $\tau_{s c}$ includes the waiting time for medium access and the transmission time of a $f_{s c}$ frame (noted $D_{s c}$ )

- The time delay due to transferring $f_{c a}$ frames (noted $\tau_{c a}$ ) elapsed from the ready-to-send instant of the $f_{c a}$ frame till the reception instant of this frame by the actuator. The time delay $\tau_{c a}$ includes the waiting time for medium access and the transmission time of a $f_{c a}$ frame (noted $\left.D_{c a}\right)$

Note that $D_{s c}$ and $D_{c a}$ can be easily calculated based on the frame length and the network bit rate.

Therefore, the communication time delay of a closed-loop control system is:

$$
\tau=\tau_{s c}+\tau_{c a}
$$

In this paper, we consider the following hypotheses:

- Communication time delay $\tau<h$.

- Computational time in the controller, sensors is neglected.

- There is no data loss.

- Clocks of the sensor and the controller are synchronized, i.e. the controller recognizes the sampling instant $t_{k}$.

\subsection{Model of NCSs under Communication Time Delay}

As shown in Fig. 3, a NCS has a continuous plant (Equation (8)) and a discrete controller (Equation (9)).

$$
\begin{gathered}
\left\{\begin{array}{c}
\dot{x}=A x(t)+B u(t) \\
y(t)=C x(t)+D u(t)
\end{array}\right. \\
u(k h)=-K_{d} x(k h), k=0,1,2 \ldots
\end{gathered}
$$

Where $x(t)$ is the state vector, $u(t)$ is the input vector and $y(t)$ is the output vector, $\mathrm{A}$ is the system matrix, $\mathrm{B}$ is the input matrix, $\mathrm{C}$ is the output matrix, $\mathrm{D}$ is the connected matrix. And $K_{d}$ is the state feedback gain matrix.

State-space model in discrete time domain with the communication time delay $\tau$ is described as follows:

$$
\left\{\begin{aligned}
x(k h+h) & =\Phi x(k h)+\Gamma_{0}(\tau) u(k h)+\Gamma_{1}(\tau) u(k h-h) \\
y(k h) & =C x(k h)+D u(k h)
\end{aligned}\right.
$$

Where $\Phi, \Gamma_{0}(\tau), \Gamma_{1}(\tau)$ are the state matrix defined as follows:

$$
\begin{gathered}
\Gamma_{0}(\tau)=\int_{0}^{h-\tau} e^{A s} d s B \\
\Gamma_{1}(\tau)=e^{A(h-\tau)} \int_{0}^{\tau} e^{A s} d s B
\end{gathered}
$$

State-space model in Equation (10) can be re-written as follows:

$$
\left[\begin{array}{c}
x(k h+h) \\
u(k h)
\end{array}\right]=\left[\begin{array}{cc}
\Phi & \Gamma_{1}(\tau) \\
0 & 0
\end{array}\right]\left[\begin{array}{c}
x(k h) \\
u(k h-h)
\end{array}\right]+\left[\begin{array}{c}
\Gamma_{0}(\tau) \\
I
\end{array}\right] u(k h)
$$

The discrete controller is:

$$
u(k h)=-K_{d}(\tau)\left[\begin{array}{c}
x(k h) \\
u(k h-h)
\end{array}\right]
$$

Note that the parameters in Equation (10) and Equation (13) depend on $h$ and $\tau$.

\subsection{Stability Analysis}

Equation (13) can be rewritten as follows:

$$
\left[\begin{array}{c}
x(k h+h) \\
u(k h)
\end{array}\right]=\left(\left[\begin{array}{cc}
\Phi & \Gamma_{1}(\tau) \\
0 & 0
\end{array}\right]-\left[\begin{array}{c}
\Gamma_{0}(\tau) \\
I
\end{array}\right] K_{d}(\tau)\right)\left[\begin{array}{c}
x(k h) \\
u(k h-h)
\end{array}\right](7)
$$

The matrix of the closed-loop control system is defined as follows:

$$
\Phi_{c l}=\left[\begin{array}{cc}
\Phi & \Gamma_{1}(\tau) \\
0 & 0
\end{array}\right]-\left[\begin{array}{c}
\Gamma_{0}(\tau) \\
I
\end{array}\right] \cdot K_{d}(\tau)
$$

With each different sample period, we will find $\Phi_{c l}$. We call $k$ is the number of sampling period; we have the following cases:

$$
\begin{aligned}
& k=1 \Rightarrow x(h)=\Phi_{c l} x(0) \\
& k=2 \Rightarrow x(h+h)=\Phi_{c l} x(h)=\Phi_{c l} \Phi_{c l} x(0)=\Phi_{c l}^{2} x(0) \\
& k=3 \Rightarrow x(2 h+h)=\Phi_{c l} x(2 h)=\Phi_{c l} \Phi_{c l}^{2} x(0)=\Phi_{c l}^{3} x(0) \\
& k=4 \Rightarrow x(3 h+h)=\Phi_{c l} x(3 h)=\Phi_{c l} \Phi_{c l}^{3} x(0)=\Phi_{c l}^{4} x(0) \\
& \ldots \\
& k \quad \Rightarrow x(k h+h)=\Phi_{c l} x(k h)=\Phi_{c l} \Phi_{c l}^{k-1} x(0)=\Phi_{c l}^{k} x(0)
\end{aligned}
$$

Thus the closed-loop matrix will be the product of the matrix elements, which are calculated as follows:

$$
\left\{\prod_{k=1}^{\infty} \Phi_{c l_{k}}\right\}
$$

Closed-loop matrix in Equation (17) is used to analyze the stability of feedback control systems.

The stability condition is that largest eigenvalue of a closed-loop matrix (Equation (17)) is smaller than 1 [8]:

$$
\left|\lambda_{\max }\left(\prod_{k=1}^{\infty} \Phi_{c l_{k}}\right)\right|<1
$$




\subsection{Global Control System which is Considered}

We now present the system which will be analyzed in this work by means of the simulator TrueTime [9], a toolbox based on Matlab/Simulink which allows to simulate real-time distributed control systems.

\subsubsection{General Considerations}

We implement 8 identical process control applications (noted $\mathrm{P}_{1}, \mathrm{P}_{2} \ldots \mathrm{P}_{8}$ ) through a CAN network (Fig. 4). So we have 24 different nodes connected through a CAN network and 16 data flows ( $8 f_{s c}$ and $8 f_{c a}$ flows) sharing the network. We consider other parameters and conditions as followed:

- Bit rate $=125 \mathrm{Kbit} / \mathrm{s}$.

- Data field length of a frame $=8$ bytes. Thus, $\mathrm{D}_{\mathrm{sc}}=\mathrm{D}_{\mathrm{ca}}=$ 150 bits [10].

- The sensor tasks are synchronous and have the same sampling period.

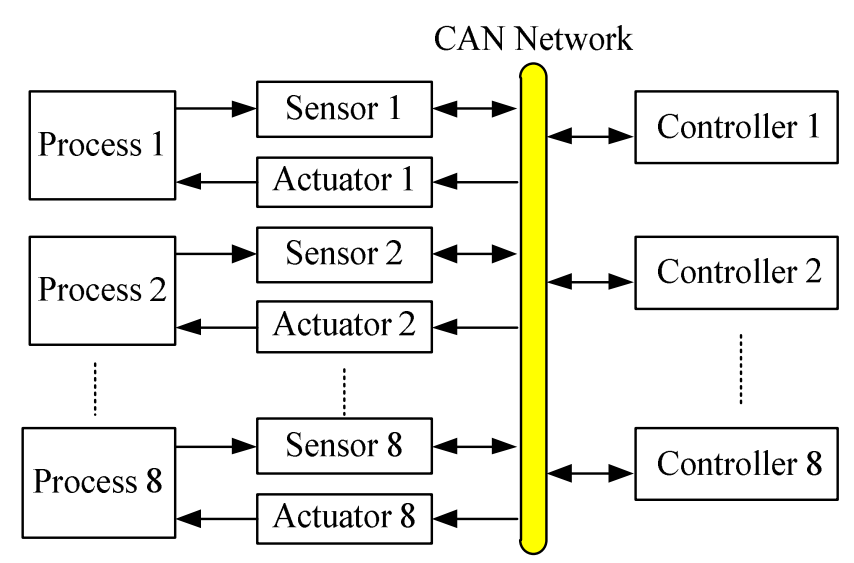

Fig 4. Implementation of 8 process control applications on the CAN network.

The scheduling of the frames is done in the MAC layer by means of priorities represented by the Identifier (ID) field of the frame.

Generally, the priorities are static priorities, i.e. each flow has a unique priority (specified a priori out of line) and all the frames of this flow have the same priority. Concerning the priorities, we will consider here either static priorities (i.e. each flow has a unique priority specified out of line) or hybrid priorities (as we said in the introduction) i.e. with two priority levels. One level represents the flow priority which is a static priority. The other level represents the frame transmission urgency. The urgency can be the same for all the frames of the flow and, in this case, the transmission urgency is also a static priority. The urgency can vary (for example, if the conditions of the application, which uses the flow, change) and, in this case, the transmission urgency is a dynamic priority. This concept has a great interest during the transient behavior of systems $[12,13]$.

The consideration of hybrid priorities requires structuring the field ID in two levels (Fig. 5) where the Level 1 represents the flow priority and the Level 2 represents the urgency priority [4].

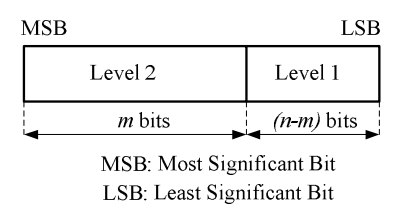

Fig 5. Structure of the ID field.

In the context of the competition based on these hybrid priorities, the frame scheduling is executed by comparing first the bits of the Level 2 (urgency predominance). If the urgencies are identical, the Level 1 (static priorities which have the uniqueness properties) resolves the competition.

\subsubsection{Static Priorities Associated to the $F_{s c}$ and $F_{c a}$ Flows}

Here we consider the conclusion shown in [11]: The priority of the $f_{c a}$ flow should be higher than the priority of the $f_{s c}$ flow in order to get the best results. Considering 8 process control applications $\left(\mathrm{P}_{1}, \mathrm{P}_{2} \ldots \mathrm{P}_{8}\right)$, each process has one $f_{s c}$ flow and one $f_{c a}$ flow. We call Prio $f_{c a} i$ and Prio $f_{s c} i$ the priorities of the $f_{s c}$ and $f_{c a}$ flows of the process $\mathrm{P}_{\mathrm{i}}(i=1,2 \ldots 8)$, respectively. The priorities of 16 data flows are arranged in the following order:

Prio $f_{\text {ca }} l>$ Prio $f_{\text {ca_ }} 2>\ldots>$ Prio $f_{\text {ca_ }} 8>$ Prio $f_{\text {sc }} l>$ Prio $f_{\text {sc }} 2>\ldots>$ Prio $f_{\text {sc }}$ 8. i.e. the controller has a higher priority than the sensor and during a sampling period, the order of medium access is arranged as $\mathrm{P}_{1}, \mathrm{P}_{2}, \ldots, \mathrm{P}_{8}$.

\subsubsection{Criteria of the QoC Evaluation}

We will consider the time response for the QoC evaluation.

\subsubsection{Stability Analysis}

As we know the lengths of frames of all processes and the order of medium access, we can calculate the communication time delay of each process. The time delays of $\mathrm{P}_{1}, \mathrm{P}_{2} \ldots \mathrm{P}_{8}$ are $2.4 \mathrm{~ms}, 4.8 \mathrm{~ms}, 7.2 \mathrm{~ms}, 9.6 \mathrm{~ms}, 12 \mathrm{~ms}, 14.4 \mathrm{~ms}$, $16.8 \mathrm{~ms}, 19.2 \mathrm{~ms}$ respectively. We consider also a time delay of $23.55 \mathrm{~ms}$.

The largest eigenvalues of the closed-loop matrix corresponding to the time delay $\tau$ above are represented in Table 1. For the case of non-compensation, the higher the delay is, the higher the $\left|\lambda_{\max }\left(\Phi_{c l}\right)\right|$ is, which is logical. For the 8 processes, the $\left|\lambda_{\max }\left(\Phi_{c l}\right)\right|<1$, thus our system is stable. We see that if the time delay is $23.55 \mathrm{~ms}$, $\left|\lambda_{\text {max }}\left(\Phi_{c l}\right)\right|=1.009>1$, the system will be unstable.

Table 1. Stability Analysis.

\begin{tabular}{lll}
\hline $\boldsymbol{\tau}$ (ms) & $\begin{array}{l}\left|\lambda_{\max }\left(\Phi_{c l}\right)\right| \\
\text { (non-compensation) }\end{array}$ & $\begin{array}{l}\left|\lambda_{\max }\left(\Phi_{c l}\right)\right| \\
\text { (compensation) }\end{array}$ \\
\hline 2.4 & 0.000598 & 0.000597 \\
4.8 & 0.000623 & 0.000597 \\
7.2 & 0.000635 & 0.000597 \\
9.6 & 0.000647 & 0.000597 \\
12 & 0.000658 & 0.000597 \\
14.4 & 0.000669 & 0.000597 \\
16.8 & 0.000679 & 0.000597 \\
19.2 & 0.0028 & 0.000597 \\
23.55 & 1.009 & 0.000597 \\
\hline
\end{tabular}


For the case of Compensation, the $\left|\lambda_{\max }\left(\Phi_{c l}\right)\right|$ is equal for all time delays. It is due to the fact that we have maintained the dominant poles, hence the closed-loop matrix has not been changed, thus the eigenvalues of the closed-loop matrix have not been changed.

\section{Proposal for Computation and Compensation of Time Delays}

\subsection{Ideas}

The goal of this subsection is to propose a way to calculate the closed-loop communication time delay and we compensate this time delay using the pole placement design method in order to improve the QoC for CAN-based NCSs. We consider the implementation of several process control applications on a CAN network. Then we show the interest of the proposed method by comparing the QoC in cases of time delay compensation and of without time delay compensation.

\subsection{Computation of Closed-Loop Communication Time Delays}

The computation of closed-loop communication time delays is done by the controller in each sampling period.

Concerning the time delay $\tau_{s c}$, as the controller has the knowledge of sampling instants $t_{k}(k=0,1 \ldots)$, it can easily deduce the value of $\tau_{s c}$ by the time difference between the reception instant of $f_{s c}$ frames and $t_{k}$.

Concerning the time delay $\tau_{c a}$, it cannot be calculated because the $f_{c a}$ frames have not been transmitted yet. However, due to the hypotheses in section 2.6 (i.e. the priority of the controller is higher than this of the sensor; there is no competition between the controllers; there is no data lost), the controller can immediately send its frame. Therefore, $\tau_{c a}$ is equal to the duration of a frame transmission $\left(D_{c a}\right)$.

The closed-loop time delay will be computed by the controller as followed:

$$
\tau=\tau_{s c}+D_{c a}
$$

\subsection{Time Delay Compensation Steps}

The compensation for time delays done by the controller in each sampling period has the following steps:

- Step 1: Identifying expected poles including the dominant poles and the other poles [7] which are selected equally to the real part of the dominant pole divided $\alpha$, with $\alpha=2 \div 10$.

- Step 2: Computing closed-loop communication time delay.

- Step 3: Computing the controller parameters according to the time delay value in order to maintain the position or the value of the expected poles.

- Step 4: Computing he control signal based on the new control parameters calculated in the previous step.

\subsection{Considering the Implementation of the 8 Process Control Applications on CAN Network}

We want to show here the interest of the pole-placement design method (which is based on an adaptive controller i.e. the parameters of the controller are modified according to the communication time delay) in comparison with the case where we without time delay compensation (i.e. we have a fixed controller).

The QoC is represented in Table $2\left(\Delta J / J_{0} \%\right)$ and Fig. 6 and Fig. 7.

Table 2. $Q \circ C\left(\Delta J / J_{0} \%\right)$.

\begin{tabular}{ccc}
\hline Process & Non-Compensation & Compensation \\
\hline $\mathrm{P}_{1}$ & 0.47 & 0.33 \\
$\mathrm{P}_{2}$ & 1.02 & 0.74 \\
$\mathrm{P}_{3}$ & 1.80 & 1.29 \\
$\mathrm{P}_{4}$ & 2.94 & 2.08 \\
$\mathrm{P}_{5}$ & 4.67 & 3.22 \\
$\mathrm{P}_{6}$ & 7.57 & 4.96 \\
$\mathrm{P}_{7}$ & 13.18 & 7.87 \\
$\mathrm{P}_{8}$ & 27.54 & 13.50 \\
\hline
\end{tabular}

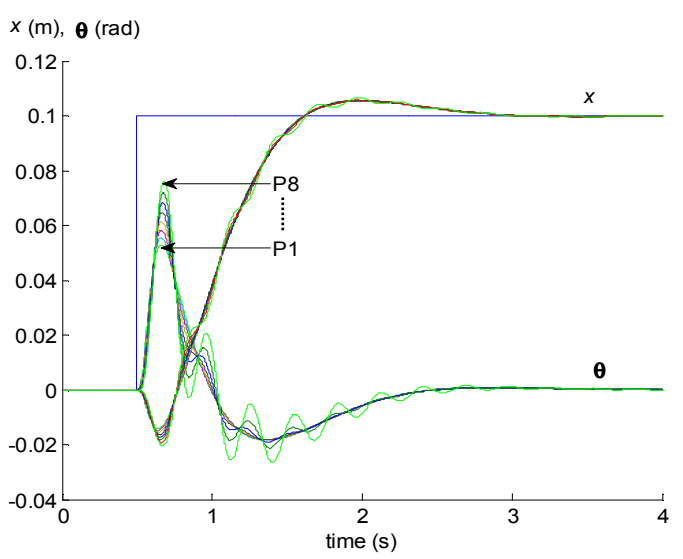

Fig 6. Time responses when non-compensation.

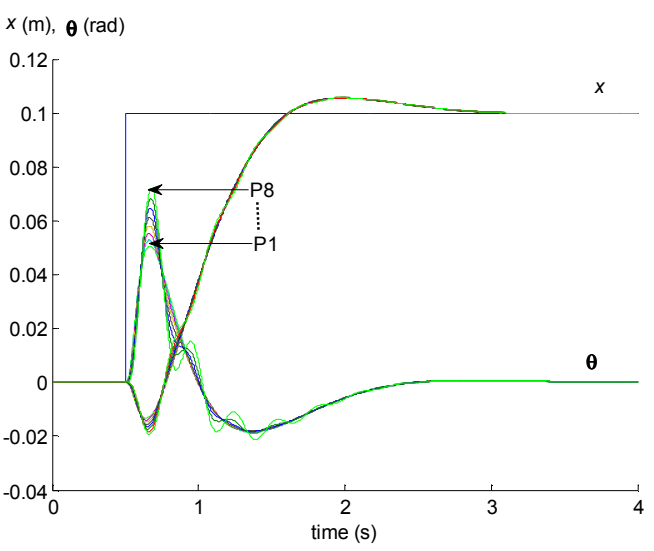

Fig 7. Time responses when compensation.

Remind that the priority of the process $\mathrm{P}_{\mathrm{i}}$ is higher than this of the process $P_{j}$. We see in Table 2 for the both cases that the QoC follows the order of the priority, it is reasonable because the higher the priority is, the lower the time delay is and so the better the QoC is. 
In the both Table 2 and Fig. $6 \& 7$, we can see the improvement of the QoC for the case of compensation compared with the case of non-compensation expressed through smaller values of $\Delta J / J_{0} \%$ and less oscillatory time responses

\section{Proposal of a Hybrid Priority Scheme for Message Scheduling}

\subsection{Limitations of Static Priorities}

Considering static priorities, each node in the network has a unique and fixed priority. Hence, the application which has data flows of low priorities could not get the desired QoS and QoC. For example, we consider a control system with two nodes A and B, in which A has higher priority than B. At the instant $t$, both A and B have frames to transmit, B has a stronger transmission urgency in order to satisfy the QoS requirement (for example the deadline) but $\mathrm{B}$ cannot transmit its frames before the end of the frame transmission of A. That will make the system less efficient.

Other limitations of static priority can be found when we consider time response of process control applications which is characterized by two regimes: transient regime (because of an input change or a noise) and permanent regime (system is in the steady state). In transient regime, frames must be transmitted as soon as possible in order to obtain some QoC requirements such as response time, overshoot, rise time (i.e. frames have high transmission urgencies). Contrariwise, in permanent regime, frames are required to be transmitted quickly (i.e. frames have low transmission urgencies). It is clearly noticed that static priority cannot overcome this problem.

\subsection{Hybrid Priorities and Related Works}

\subsubsection{Idea of Hybrid Priorities}

The idea of hybrid priority results from the limitations of static priority presented in the previous section. The idea is that frame priorities can vary depending on transmission urgency. That can be done by restructuring the ID field into 2 small fields as represented in Fig. 5.

Level 2 ( $m$ high significant bits) represents transmission urgency which is called dynamic priority part with its value $I D \_d y n$. The ID_dyn can be changed during system operation and several data flows can share the same $I D \_d y n$. Level 1 ( $n-m$ bits) which is called static priority part represents the uniqueness of data flows as its value $I D \_s t a$ is fixed, unique and specified before system running. The uniqueness means that there are no two or more nodes having the same ID_sta. The term "hybrid priority" means the combination of dynamic priority and static priority.

The idea of this ID field structure was first introduced in [12]. Then other studies $[3,14,15]$ also used the similar ID field structure.

The medium access tournament is done firstly by comparing Level 2. If there are several data flows having the same ID_dyn, Level 1 will determine the only winner allowed to access to the medium.

Remark: With $(n-m) I D \_s t a$ bits, we can determine $2^{\text {n-m }}$ data flows (or nodes) sharing the network. Therefore, it must be careful when we choose the number of ID_sta bits.

\subsubsection{Specifying of the Dynamic Priority}

Specifying dynamic priority part requires, firstly, to determine QoC parameter of the process control application which gives information on the transmission urgency, and secondly, to translate these urgencies into dynamic priorities (i.e. computation of dynamic priorities).

Two main QoC parameters using for representing the transmission urgency are steady state error $e[3,14]$ and control signal $u[4,15]$. Some other works use the deadline $[12,13]$. With these parameters, the authors proposed different functions for computation of dynamic priorities. The principle is that the higher the values of $e, u$ or deadline are, the higher the dynamic priorities are.

Concerning the works using the error $e$, they used an extended ID field of 29 bits (16 bits for ID_dyn and 10 bits for ID_sta). The value of $e$ is encoded directly into the ID_dyn value. The first limitation is that, we have a wide range of error value and this is not bounded (for example when the system is unstable, the error is infinite). Mapping these error values in a definite number of priority bits is not an easy task. The second limitation is that, they assume the existence of a master node knowing the current states of all controller nodes. Maintaining a global state in the whole distributed system can be problematic.

The works using the control signal $u$ have overcome the unbounded value by a saturation value us (if $u$ is higher than $u_{s}$, the dynamic priority is maximum). They used a standard ID filed of 11 bits ( 7 bits for ID_dyn and 4 bits for ID_sta). So, they can determine a maximum number of 16 data flows (or nodes) which is not enough to address all nodes in a NCS.

\subsection{Proposal}

\subsubsection{Distributed Aspect}

The NCS is totally distributed, i.e. there is not any master node.

\subsubsection{ID Field}

We use an extended ID field of 29 bits with 11 bits for dynamic priority part, and 11 bits for static priority part. That overcomes the limitation concerning number of $I D \_s t a$ bits.

\subsubsection{Control Parameters}

Both the error $e$ and the control signal $u$ are used for making the dynamic priority.

\subsubsection{Computation of Dynamic Priorities}

The dynamic priority (noted Prio_dyn) is calculated by the controller using functions represented in Fig. 8 and equations (20) \& (21). Here, we consider that $e_{s}$ and $u_{s}$ are the maximum values of $e$ and $u$ respectively when we 
consider the initial continuous control system without the network.

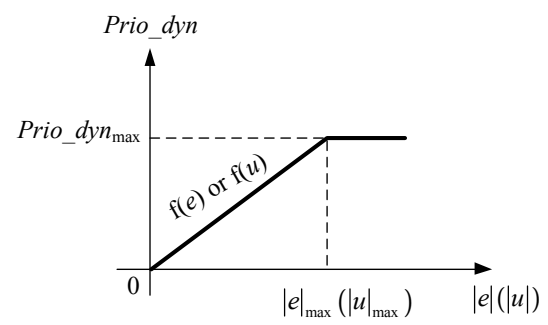

Fig 8. Functions of e, $u$.

$$
\begin{aligned}
& f(e)= \begin{cases}\text { Prio_dyn } n_{\text {max }} \frac{|e|}{|e|_{\text {max }}}, & 0 \leq|e| \leq|e|_{\text {max }} \\
\text { Prio_dyn } n_{\text {max }}, & |e|>|e|_{\text {max }}\end{cases} \\
& f(u)= \begin{cases}\text { Prio_dyn } n_{\text {max }} \frac{|u|}{|u|_{\text {max }}}, & 0 \leq|u| \leq|u|_{\text {max }} \\
\text { Prio_dyn } \text { max }_{\text {max }}, & |u|>u_{\text {max }}\end{cases}
\end{aligned}
$$

\subsubsection{Encoding Priority into ID Field}

The dynamic priority part consists of 11 bits which is able to represent $2^{11}=2048$ priority levels from 0 to 2047 . The minimum dynamic priority is Prio_dyn $n_{\min }=0$ corresponding to the $I D \_d y n$ of 11 recessive bits (bit 1 ).
The maximum dynamic priority is Prio_dyn $n_{\max }=2047$ corresponding to the $I D \_d y n$ field of 11 dominant bits (bit 0 ). The relation between the $I D \_d y n$ and the Prio_dyn is as follows:

$$
I D \_d y n=2047-\text { Prio_dyn }
$$

\subsubsection{Implementation of the Hybrid Priority Scheme}

Before we present how the hybrid priority scheme works, we should consider the implementation of a process control application on the CAN network as represented on Fig. 9.

We can see how the hybrid priority works. Firstly, concerning the static priority part, this priority of each node is specified before the system running. The subsection 2.6 shows that we have to set static priority of the $f_{c a}$ flow (noted Prio_sta $a_{f c a}$ ) higher than that of the $f_{s c}$ flow (noted Prio_sta $t_{f c}$ ) in order to get the best results. Here we will consider this conclusion. Secondly, concerning dynamic priority part, its implementation is as follows:

- At the instant $t_{k}$, the sensor samples the output $\left(y_{k}\right)$ and gets dynamic priority (Prio_dyn $n_{k-1}$ ) sent from the controller in the previous period (i.e. period starting at $\left.t_{k-1}\right)$. After that, the sensor uses this priority $\left(\right.$ Prio_ $\left.d y n_{k-1}\right)$ to send its frame (containing $y_{k}$ ) to the controller.

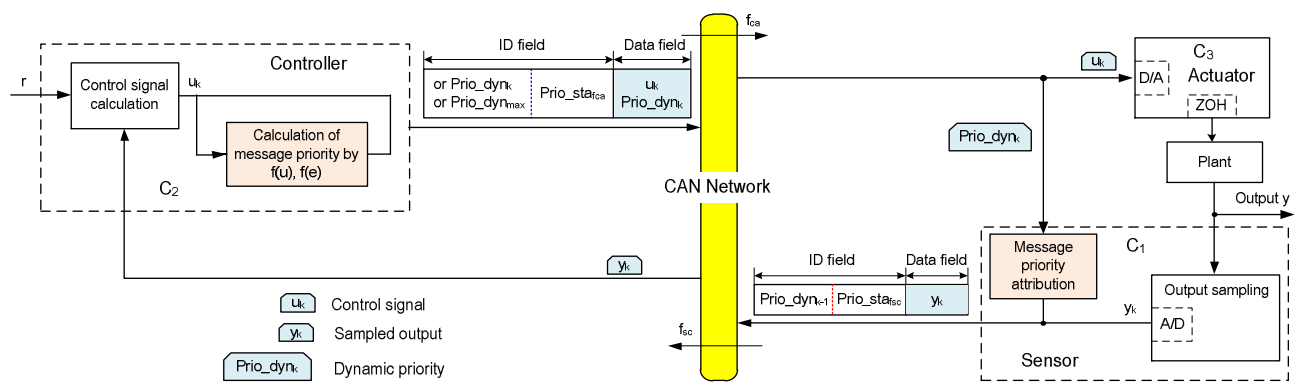

Fig 9. Implementations of process control applications on a CAN network.

Table 3. $\operatorname{QoS}(\bar{\tau} \mathrm{ms})$.

\begin{tabular}{cccccccccc}
\hline & $\mathbf{P}_{\mathbf{1}}$ & $\mathbf{P}_{\mathbf{2}}$ & $\mathbf{P}_{\mathbf{3}}$ & $\mathbf{P}_{\mathbf{4}}$ & $\mathbf{P}_{\mathbf{5}}$ & $\mathbf{P}_{\mathbf{6}}$ & $\mathbf{P}_{\mathbf{7}}$ & $\mathbf{P}_{\mathbf{8}}$ & $\bar{\tau}_{\text {max }}-\bar{\tau}_{\text {min }}$ \\
\hline Static priority & 2.4 & 4.8 & 7.2 & 9.6 & 12 & 14.4 & 16.8 & 19.2 & 16.8 \\
Hybrid priority with $e$ & 5.9 & 7.7 & 9.2 & 10.4 & 13.7 & 12.3 & 14.4 & 10.3 & 8.5 \\
Hybrid priority with $u$ & 9.5 & 9.9 & 11.0 & 10.4 & 12 & 10.2 & 12.6 & 12.9 & 3.4 \\
\hline
\end{tabular}
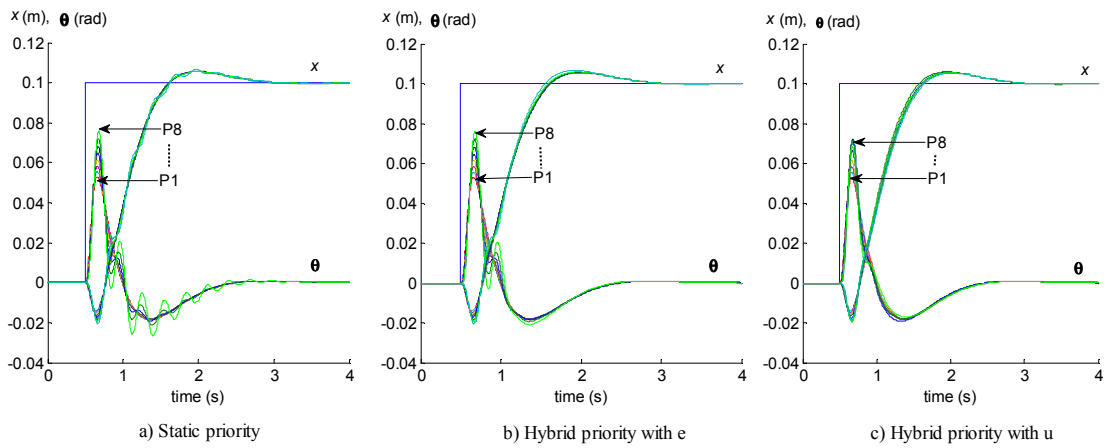

Fig 10. Time responses when non-compensation. 
- After receiving the $f_{s c}$ frame sent from the sensor, the controller computes the control signal $u_{k}$ and the dynamic priority Prio_dyn $n_{k}$ (by equations (20) \& (21)) and sends its frame on the network. Then, the actuator will get $u_{k}$ and apply it to the controlled plant, while the sensor will get Prio_dyn $n_{k}$ to use in the next sampling period (period starting at $t_{k+1}$ ).

Concerning dynamic priority using to send the $f_{c a}$ frame by the controller, there are two ways: the first one is that the controller use the Prio_dyn $n_{k}$ value which has just been computed [15]; and the second one is to use the Prio_dyn $n_{\max }$ [4]. It is evident that the second way ensures that $f_{c a}$ frame will be sent immediately after the reception of $f_{s c}$ frame (computational time delays in the controller is negligible). Therefore, comparing to the first way, the second way performs a shorter time delay of the closed loop. In this paper, we consider the second one, i.e. the controller uses the Prio_dyn $n_{\max }$ to send its frames.

Noting that, at the instant $0\left(t_{0}=0\right)$, the sensor has no information about the dynamic priority from the controller. Therefore we consider that the sensor uses, at the first time, the Prio_dyn $n_{\max }$.

\subsection{Implementation of Process Control Application on CAN Network}

\subsubsection{Communication Time Delay}

These time delays are network delays in the communication between the $f_{s c}$ frame (noted $\tau_{s c}$ ) and the $f_{c a}$ frame (noted $\tau_{c a}$ ). During each sampling period, time delay $\tau_{s c}$ is the time difference between sampling instant and reception instant of the $f_{s c}$ frame by the controller; the time delay $\tau_{c a}$ is the time elapsed from the ready-to-send instant of the $f_{c a}$ frame till the reception instant of this frame by the actuator. Therefore, communication delay in the sampling period is computed as follows:

$$
\tau=\tau_{s c}+\tau_{c a}
$$

\subsubsection{Criteria of the QoS Evaluation}

In order to evaluate the QoS, we calculate first the communication time delay $\tau_{i}$ of the closed loop control system in each sampling period starting at $t_{i}$ according to the equation (23), then we compute the average value of theses time delays during the settling time $t_{s}$ by the following formula:

$$
\bar{\tau}=\frac{1}{n} \sum_{i=1}^{n} \tau_{i}
$$

Where $n$ is the number of sampling period in the settling time.

The smaller the value $\bar{\tau}$ is, the better the QoS is.

\subsubsection{Criteria of the QoC Evaluation}

The QoC is considered through the time responses.
4.4.4. Results
a. Quality of Service

We present on the Table 3 the QoS in term of $\tau$ of the 8 processes.

For static priority scheme, the process with higher priority has smaller time delay. We see that $\mathrm{P}_{1}$ has the highest priority so its delay is the smallest while $\mathrm{P}_{8}$ has the lowest priority so its delay is the biggest. It is logical.

For the hybrid priority scheme (with $e$ and $u$ ), we obtain time delays more balanced than these with static priorities. This is the result of the predominant role of the parts "dynamic priority" compared with the parts "static priority".

The QoS balance can be observed by the difference between the maximum delay and the minimum delay in each priority scheme in the Table 3 . We see that these differences are small with hybrid priorities ( $8.5 \mathrm{~ms}$ with $e$ and $3.4 \mathrm{~ms}$ with $u$ ) while this value is very big with static priorities $(16.8 \mathrm{~ms})$.

\section{b. Quality of Control}

The QoC is represented in Fig. 10 (time responses). We see also the balances of QoC with hybrid priorities compared with static priorities. It is logical because balances of QoS induce balances of QoC. The conclusions of QoC for different priority schemes are similar to those of QoS. Precisely, for static priority scheme, the higher the priority is, the better the QoC is. And for hybrid priority schemes, the QoCs are more balanced than that of the static priority scheme.

\section{Co-Design of Compensation for Communication Time Delays and the Message Scheduling}

\subsection{Ideas}

The idea is to combine the frame scheduling scheme based on the hybrid priority and the method of compensation for communication time delay in order to have a more efficient NCSs. However, concerning the close-loop time delay compensation, in the sampling period $k$, we cannot consider here that the controller can use the value of the close-loop time delay of the sampling period $(k-1)$ because now, taking into account for the dynamic priority used by the sensor task, the time delay $\left(\tau_{\mathrm{sc}}+\tau_{\mathrm{ca}}\right)$ changes every period. Then the controller must make the delay compensation in the sampling period $k$ by knowing the close-loop time delay of this sampling period $k$. We explain now this implementation.

\subsection{Principle of the Implementation of the Co-Design}

This principle, relatively to the sampling period starting at $t_{k}$, is represented on Fig. 11 where we indicate the content of the $f_{s c}$ and $f_{c a}$ frames and the computations done by the controller. The process of co-design implementation in each cycle starting at $t_{k}$ as follows:

Sensor sampling state variables $x$ and receive priority value sent from the controller in the previous period (period starting at $\left.t_{k-1}\right)$, then use the sensor priority this (Prio_dyn $\left.n_{k-1}\right)$ 
to send a message containing $x_{k}$ to the controller.

- The controller after receiving the signal from the sensor $x_{k}$, will perform the following steps:

- The Computations of dynamic priority Prio_dyn $n_{k}$ based on the function $f(u)$ and $f(e)$ (by equations (20) \& (21)).

- Calculations of close-loop communication time delay $\tau$ and the parameters of the controller according to the communication time delay $\tau$, i.e. implementation of the compensation for close-loop communication time delay.

- Calculations of the control signal $u_{k}$.

- Send messages including control signal $u_{k}$ and dynamic priority Prio_dyn $n_{k}$ on the network.

Then the actuator will receive the value $u_{k}$ and apply to control plant, while the sensor will get Prio_dyn $n_{k}$ to use for the next period (period starting at $t_{k+1}$ ).

\subsection{Performance Evaluation and Summary of Obtained Results}

We still consider the implementation of 8 applications $\left(\mathrm{P}_{1}\right.$, $\left.\mathrm{P}_{2}, \ldots, \mathrm{P}_{8}\right)$ studied in the previous sections.

The time responses are represented on Fig. 12. These results which are represented graphically on obviously show the balanced performances provided by the hybrid priority compared with the static priority and by the co-design compared with the static priority scheme (compensation).

From these results, we can say that, if we have a constraint of performance which cannot be exceeded (not too small), the hybrid priority allows to implement more applications than the static one and, extensionally, the bidirectional relation co-design allows to implement more applications than the static priority scheme (compensation).

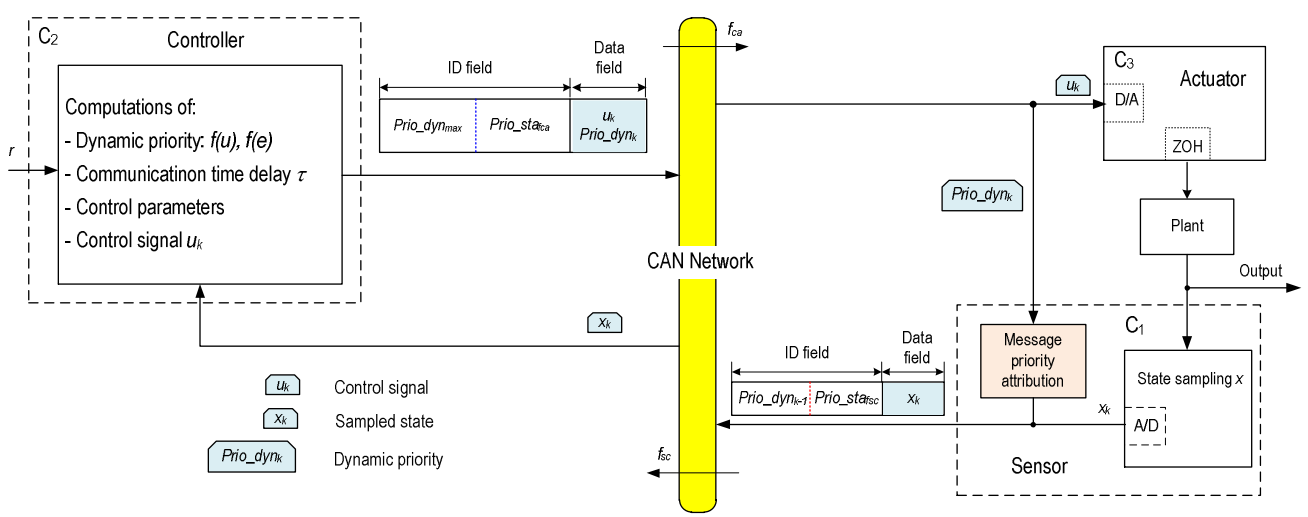

Fig 11. Principle of the implementation of the co-design.

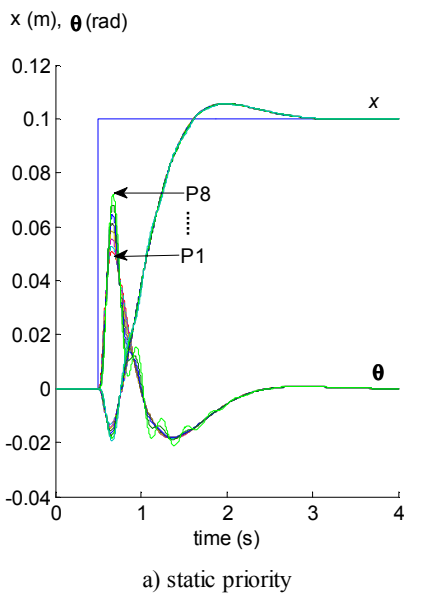

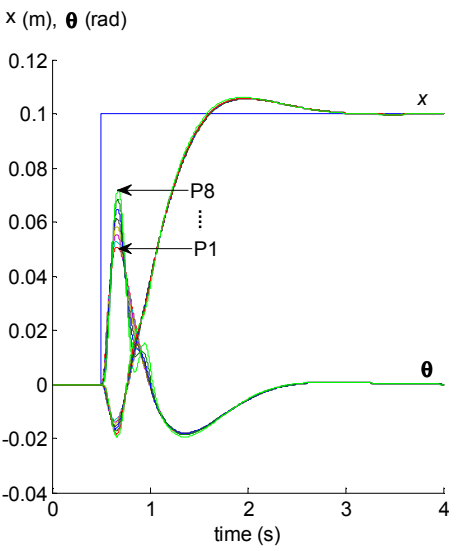

b) hybrid priority with e

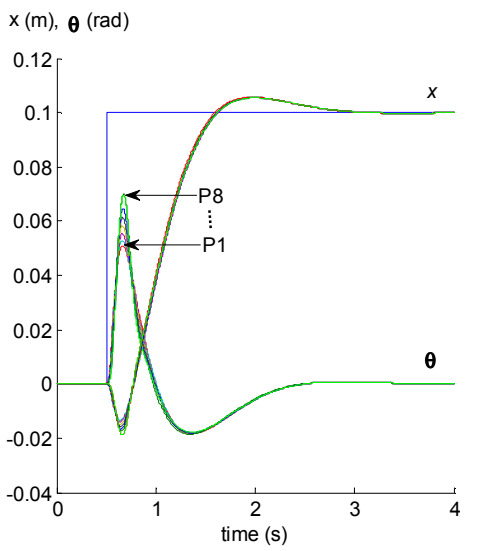

c) hybrid priority with $\mathrm{u}$

Fig 12. Time responses when compensation.

\section{Conclusion}

In this paper, we have presented three following points:

- The first is the implementation of the way to calculate the closed-loop communication time delay and we compensate this time delay using the pole placement design method in order to improve the QoC performances provided by static priorities only.

- The second is the implementation of the hybrid priority scheme for the message scheduling in order to improve the QoS. The hybrid priority is characterized by, on one hand, a static part representing the uniqueness of the flow, and on the other hand, a dynamic part that represents the urgency of transmission (this dynamic part is expressed from a function of the error and control signal). This hybrid priority gives, in comparison to the static priority case, balanced performances for the different process control applications.

- The final is the implementation of the bidirectional 
relation QoS and QoC (i.e. co-design) which is the combination of the between conpensation for communication time delay and the message scheduling in order to have a more efficient NCSs design. the relation. The results, which are obtained, show the interest of the joint action of the hybrid priorities and the delay compensation (by the hybrid priorities, we introduce the balance aspect compared with the static priority; by the delay compensation, we maintain the balance aspect while improving the QoC and then we can consider the possibility to implement more applications than with the static priority scheme (compensation).

The further work should be the following points: the utilization of other compensation methods for time delays (for example, maintenance of the phase margin); the consideration of other types of controller (PID for example) and the consideration of other types of process control applications. Still furthermore, the study of this relation might be also improved by the consideration of theoretical problems (in particulars, stability conditions when the online control law parameters change from sampling period to sampling period).

\section{References}

[1] Michael S. Branicky, Vincenzo Liberatore and Stephen M. Phillips, "Networked control system co-simulation for co-design," American Control Conference, USA, Vol. 4, June 2003, pp. 3341-3346.

[2] Ye-Qiong Song, "Networked Control Systems: From independent designs of the network QoS and the control of the co-design," 8th IFAC international Conference on Fieldbuses and Networks in Industrial and Embedded Systems, Korea, May 2009, pp. 155-162.

[3] Pau Martí, José Yépez, Manel Velasco, Ricard Villà and Josep M. Fuertes, "Managing Quality-of-Control in network-based control systems by controller and message scheduling co-design," IEEE Transactions on Industrial Electronics, Vol. 51, No. 6, Dec. 2004, pp. 1159-1167.

[4] Xuan Hung Nguyen and Guy Juanole, "Design of Networked Control Systems on the basis of interplays between Quality of Control and Quality of Service," 7th IEEE International Symposium on Industrial Embedded Systems, France, June 2012, pp. 85-93.

[5] Y.B. Zhao, G.P. Liu and D. Rees, "Integrated predictive control and scheduling co-design for networked control systems," IET Control Theory \& Applications, Vol. 2, Issue 1, Jan. 2008, pp. 7-15.

[6] Shi-Lu Dai, Hai Lin, and Shuzhi Sam Ge, "Scheduling and control co-design for a collection of Networked Control Systems with uncertain delays," IEEE Transactions on control systems technology, Vol. 18, No. 1, Jan. 2010, pp. 66-78.

[7] Karl J. Åström and B. Wittenmark, "Computer controlled systems: theory and design,” 3th Edition, Prentice Hall, 1997.

[8] Murat Dogruel and Umit Özgüner, "Stability of a Set of Matrices-A Control heoretic Approach," 34th Conference on Decision and Control, New Orleans, USA, Vol. 2, Sep. 1995, pp. 1324-1329.

[9] Martin Ohlin, Dan Henrikssonand Anton Cervin, "TrueTime 1.5 - Reference Manual," Lund Institute of Technology, Sweden, 2007.

[10] Salem Hasnaoui, Oussema Kallel, Ridha Kbaier, Samir Ben Ahmed, "An implementation of a proposed modification of CAN protocol on CAN fieldbus controller component for supporting a dynamic priority policy," 38th Annual Meeting of the Ind. App., Vol. 1, Oct. 2003, pp. 23-31.

[11] Guy Juanole, Gerard Mouney, Christophe Calmettes, Marek Peca, "Fundamental considerations for implementing control systems on a CAN network," 6th International Conference on Fielbus Systems and their Applications, Mexico, Nov. 2005, pp. 280-285.

[12] Khawar M. Zuberi end Kang G. Shin, "Scheduling messages on Controller Area Network for real time CIM applications," IEEE Trans. Robot. Autom, Vol. 13, No. 2, Apr. 1997, pp. 310-314.

[13] Khawar M. Zuberi and Kang G. Shin, "Design and implementation of efficient message scheduling for Controller Area Network," IEEE Transactions on Computers, Vol. 49, No. 2, Feb. 2000, pp. 182-188.

[14] Manel Velasco, Pau Martí, Rosa Castané, Josep Guardia and Josep M. Fuertes, "A CAN application profile for control optimization in Networked Embedded Systems," 32nd Annual Conference onIEEE Industrial Electronics, Paris, Nov. 2006, pp. 4638-4643.

[15] Guy Juanole and Gérard Mouney, "Networked Control Systems: Definition and analysis of a hybrid priority scheme for the message scheduling," 13th IEEE conference on Embedded and Real-Time Computing Systems and Applications, Korea, Aug. 2007, pp. 267-274. 